\title{
Research on Fault Prediction Model and Algorithm of Communication System Based on HMM
}

\author{
Xiaotao $\mathrm{Xu}^{*}$, Yichen Song and Yonghong Gao \\ National Defense Technology University Information Communication Academy, Wuhan, China \\ ${ }^{*}$ Corresponding author
}

\begin{abstract}
The hidden model is a typical state prediction model, which has been widely used in the field of industrial production state monitoring in recent years. This paper analyzes the basic principle of HMM, puts forward three basic algorithms for fault prediction of communication system, analyzes the structural properties of the algorithm, puts forward the application model architecture of HMM, and provides the theoretical basis for constructing the fault prediction system based on HMM model.
\end{abstract}

Keywords-hidden model; communication system; fault prediction; algorithm

\section{INTRODUCTION}

As a statistical model of signal dynamic time series, Hidden Model(HMM) is very suitable to deal with continuous dynamic signals, and it has solid theoretical foundation, learning function and adaptive ability, and can acquire knowledge to monitor system state through training. The HMM, proposed by Baum and others in the late 1960s, developed gradually in the mid-1980s and began to be applied to signal processing and so on. In recent years, many scholars devoted to Hidden Model in the industrial field of state monitoring and fault classification, have achieved good results. In view of the similar properties of communication system and industrial state monitoring system, we can try to use HMM in state monitoring and health management of communication system.

\section{The APPLICATION PRINCIPLE OF HMM}

HMM is a probabilistic model describing the statistical characteristics of stochastic processes. It is developed from chain. chain is a random process of discrete variables. At different times, the system is only in one state, each state corresponds to an observation value, the transition between States has the corresponding transfer probability, and the state transfer probability only depends on the current state, independent of the past state. Unlike the chain, HMM is a double random process, one is a chain with a finite state, describing the transfer of state, and another describes the statistical correspondence between each state and observed values. Not only is the transfer between states random, but the observation value of each state is also random. You can only see the observation value from the observer's point of view, not directly, but you can perceive the state through a random process
The Hidden Model mainly solves three kinds of problems. One is to assess the problem. Solve the size of the probability of producing a specific observation sequence by a HMM, that is, given the model $\lambda$ and the observation sequence $O$, the calculation probability $P(O \mid \lambda)$ is usually calculated by the forward-backward algorithm. The second problem is decoding. For a given model $\lambda$ and an observed sequence $O$, the most likely state sequence $Q$ is usually implemented by Viterbi algorithm. Third is the question of study. Solve the problem of how to learn a parameter of HMM by a set of observation sequences in the maximum likelihood sense, namely the training of model. For a given observation sequence $O$, learn to get the model $\lambda=(\pi, A, B)$ to $P(O \mid \lambda)$ the maximum, usually trained by Baum-Welch algorithm.

\section{BASIC ALGORITHM OF HMM}

The assessment, decoding and learning problems described above can be addressed using forward-backward, Viterbi and Baum-Welch algorithms, respectively.

\section{A. Forward-backward Algorithm}

First, confirm that you have the correct template for your paper size. This template has been tailored for output on the US-letter paper size. If you are using A4-sized paper, please close this file and download the file for "MSW_A4_format".

For a fixed state sequence $Q=\left\{q_{1}, q_{2}, \cdots, q_{T}\right\}$ could be described as follow:

$$
\begin{gathered}
P(O \mid Q, \lambda)=\prod_{i=1}^{T} P\left(o_{i} \mid q_{i}, \lambda\right)=b_{q_{1}}\left(o_{1}\right) b_{q_{2}}\left(o_{2}\right) \cdots b_{q_{T}}\left(o_{T}\right) \\
(2-5) \\
b_{q_{t}}\left(o_{t}\right)=b_{j k} \mid q_{t}=S_{j}, o_{t}=V_{k} 1 \leq t \leq T .
\end{gathered}
$$

For given $\lambda$, probability of generating $Q$ could be described as follow: 


$$
P(Q \mid \lambda)=\pi_{q_{1}} a_{q_{1} q_{2}} a_{q_{2} q_{3}} \cdots a_{q_{T-1} q_{T}}
$$

The calculated probability is:

$$
\begin{aligned}
P(O \mid \lambda) & =\sum_{i=1}^{T} P\left(o_{i} \mid Q, \lambda\right) P(Q \mid \lambda) \\
& =\sum_{q_{1}, q_{2}, \cdots, q_{T}} \pi_{q_{1}} b_{q_{1}}\left(o_{1}\right) a_{q_{1} q_{2}} b_{q_{2}}\left(o_{2}\right) \cdots a_{q_{T-1} q_{T}} b_{q_{T}}\left(o_{T}\right)
\end{aligned}
$$

The calculation of the above equation increases exponentially by an order of $2 T N^{T}$ magnitude. Therefore, this method can not be used to solve the $P(O \mid \lambda)$, so Baum proposed forward-back algorithm.

1) Forward algorithm: The forward variable could be definite as follow:

$$
\alpha(i)=P\left(o_{1}, o_{2}, \cdots o_{t}, q_{t}=S_{t} \mid \lambda\right) 1 \leq t \leq T
$$

This can be done in three procedures:

Firstly is initialization, could be described as follow:

$$
\alpha(i)=\pi_{i} b_{i}\left(o_{1}\right) 1 \leq i \leq N
$$

Secondly is recursive process, could be described as follow:

$\alpha_{t+1}(j)=\left[\sum_{i=1}^{N} \alpha_{t(i)} a_{i j}\right] b_{j}\left(o_{t+1}\right) \quad 1 \leq t \leq T-1,1 \leq j \leq N$

Thirdly is end, could be described as follow:

$$
\begin{aligned}
P(O \mid \lambda) & =\sum_{i=1}^{N} \alpha_{T}(i) \\
\text { Then } b_{j}\left(o_{t+1}\right)=b_{j k} \mid o_{t+1} & =V_{k}
\end{aligned}
$$

The forward algorithm makes the computation become $N(N+1)(T-1)+N$ $N(N-1)(T-1)$ subaddition, and greatly reduces the computation.

2) Backward algorithm: The backward variable could be definite as follow:

$$
\begin{aligned}
& \beta_{t}(i)=P\left(o_{t+1}, o_{t+2}, \cdots, o_{T} \mid q_{t}=\theta_{t}, \lambda\right) 1 \leq t \leq T-1 \\
& \text { Then } \beta_{T}(i)=1
\end{aligned}
$$

This also can be done in three procedures:

Firstly is initialization, could be described as follow:

$$
\beta_{T}(i)=1_{1 \leq i \leq N}
$$

Secondly is recursive process, could be described as follow:

$$
\begin{gathered}
\beta_{t}(i)=\sum_{j=1}^{N} a_{i j} b_{j}\left(o_{t+1}\right) \beta_{t+1}(j) \\
t \leq T-1, T-2, \cdots 1, \quad 1 \leq j \leq N
\end{gathered}
$$

Thirdly is end, could be described as follow:

$$
P(O \mid \lambda)=\sum_{i=1}^{N} \beta_{1}(i)
$$

The computation of the backward algorithm is about order of $N^{2} T$ magnitude.

\section{B. Viterb Algorithm}

Set $\delta_{t}(i)$ to time $\mathrm{T}$ along a path $q_{1}, q_{2}, \cdots, q_{t}$, and $q_{t}=\theta_{t}$, then could generate the maximum probability of $q_{1}, q_{2}, \cdots, q_{t}$ is:

$\delta_{t}(i)=\max P\left(q_{1}, q_{2}, \cdots, q_{t}, q_{t}=\theta_{t}, o_{1}, o_{2}, \cdots o_{t} \mid \lambda\right)$

The best state sequence $Q^{*}$ is obtained by three procedures: Firstly is initialization, could be described as follow:

$$
\begin{cases}\delta_{i}(i)=\pi_{i} b_{i}\left(O_{1}\right) & 1 \leq i \leq N \\ \varphi(i)=0 & 1 \leq i \leq N\end{cases}
$$

Secondly is recursive process, could be described as follow:

$$
\begin{cases}\delta_{t}(j)=\max _{1 \leq i \leq N}\left[\delta_{t-1}(i) a_{i j}\right] b_{j}\left(O_{t}\right) & 2 \leq t \leq T, 1 \leq j \leq N \\ \varphi_{t}(j)=\underset{1 \leq i \leq N}{\arg \max }\left[\delta_{t-1}(i) a_{i j}\right] & 2 \leq t \leq T, 1 \leq j \leq N\end{cases}
$$

Thirdly is end, could be described as follow:

$$
\left\{\begin{array}{l}
P^{*}=\max _{1 \leq i \leq N}\left[\delta_{T}(i)\right] \\
{q_{T}^{*}}^{*}=\operatorname{Aarg} \max _{1 \leq i \leq N}\left[\delta_{T}(i)\right]
\end{array}\right.
$$


The order of status obtained is as follows:

$$
q_{t}^{*}=\varphi_{t+1}\left(q_{t+1}^{*}\right) \quad t=T-1, T-2, \cdots 1
$$

The recursion process is the most important part. When the optimal state sequence $q_{1}, q_{2}, \cdots, q_{t}$ is given from Observation value set $O_{1}, O_{2}, \ldots,{ }_{t}$, it is used to find the optimal state sequence when the part observation sequence $q_{1}, q_{2}, \cdots, q_{t}, q_{t+1}$ adds a moment. As a result, the following approximation $P\left(Q^{*} \mid O, \lambda\right) \gg \sum_{\forall Q \neq Q^{*}} P(Q \mid O, \lambda)$

is established:

$$
P^{*} \approx \sum_{\forall Q} P(Q \mid O, \lambda)
$$

Therefore, the Viterbi algorithm can also approximate calculation $P(O \mid \lambda)$.

\section{Baum-Welch Algorithm}

The algorithm mainly solves the training of HMM parameters by observation sequence, making the maximum $P(O \mid \lambda)$. The idea of Baum-Welch algorithm is to give an initial model $\lambda_{0}$, could obtain $P\left(O \mid \lambda_{0}\right)$ by the forward-backward algorithm and the observation sequence $O$ based on $\lambda_{0}$, then to get the new model $\lambda_{\text {.If }} P(O \mid \lambda)-P\left(O \mid \lambda_{0}\right)<$ parameters by maximizing the expected value, indicates that the training has achieved the desired effect, the algorithm ends. Otherwise, $\lambda=\lambda_{0}$, the repetitive training will continue until it converges. The algorithm is described in detail as follows:

Derived from the previously defined forward and backward variables as follow:

$$
P(O \mid \lambda)=\sum_{i=1}^{N} \sum_{j=1}^{N} \alpha_{t}(i) a_{i j} b_{j}\left(o_{t+1}\right) \beta_{t+1}(j)_{1 \leq t \leq T-1}
$$

Due to the limited training sequence given, the best method for parameter finding can not be obtained. Baum-Welch takes advantage of recursive processes to get bigger $P(O \mid \lambda)$, and get the best parameters.

Given training $O$ and models $\lambda$ The probability that the chain is in a $S_{i}$ state and is in a state $S_{j}$ at a time $t+1$, $\xi_{t}(i, j)$ could be definite as follow:

$$
\xi_{t}(i, j)=P\left(O, q_{t}=S_{i}, q_{t+1}=S_{j} \mid \lambda\right)
$$

This leads to:

$$
\xi_{t}(i, j)=\left[\alpha_{t}(i) a_{i j} b_{j}\left(O_{t+1}\right) \beta_{t+1}(j)\right] / P(O \mid \lambda)
$$

Could also leads to:

$$
\xi_{t}(i)=P\left(O, q_{t}=S_{i} \mid \lambda\right)=\sum_{j=1}^{N} \xi_{t}(i, j)=\left[\alpha_{t}(i) \beta_{t}(i)\right] / P(O \mid \lambda)
$$

Then the expectation of the number of times the state $S_{i}$ is transferred could be described $\sum_{t=1}^{T-1} \xi_{t}(i)$, and the expectation of the number of times the state is transferred from $S_{i}$ to $S_{j}$, could be described $\sum_{t=1}^{T-1} \xi_{t}(i, j)$. The Baum-Welch revaluation formula follows:

$$
\left\{\begin{array}{l}
\hat{a}_{i j}=\sum_{t=1}^{T-1} \xi_{t}(i, j) / \sum_{t=1}^{T-1} \xi_{t}(i) \\
\hat{b}_{j k}=\sum_{\substack{t=1 \\
o_{t}=V_{k}}}^{T-1} \xi_{t}(i, j) / \sum_{t=1}^{T-1} \xi_{t}(j) \\
\hat{\pi}_{i}=\xi_{1}(j)
\end{array}\right.
$$

Model $\lambda=(\pi, A, B)$ update to $\hat{\lambda}=(\hat{\pi}, \widehat{A}, \widehat{B})$. Baum proved $P(O \mid \hat{\lambda}) \geq P(O \mid \lambda)$ that the revalued parameters can better describe the observation sequence $O$, from which time to train until the convergence condition is met, the best model parameters of HMM are obtained.

\section{ANALySis of FAUlt ForeCASt MODEL OF COMMUNICATION SYSTEM BASED ON HMM}

In the process of state monitoring and diagnosis of communication system, the state of the system is very difficult to be directly observed. What can be detected is often some signals symptoms corresponding to the state of the communication system.

Usually in the state diagnosis of communication system, the state can be divided into two categories, that is, positive normality and failure state. In fact, the state of most communication systems from normal to fault is a slow process, its health condition is gradually declining. If the change of the fault can be studied in detail and given the corresponding intermediate state, the state of communication systems can be 
more truly reflected. So between normal state and fault state, there can also be a breakdown of the intermediate fault state. These intermediate faults can not be directly observed and need to be inferred from the observed symptoms.

The fault degree of the communication system is that the change from normal to fault state is irreversible with the deepening of the time of use. Therefore, HMM of left and right type is used to monitor and diagnose the state of the communication system. The application model could be described as figure1.

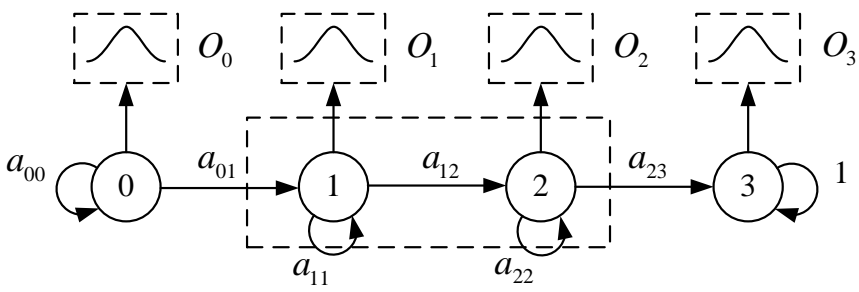

FIGURE I. APPLICATION MODEL STRUCTURE OF COMMUNICATION SYSTEM FAULT PREDICTION

In Figure 1, the state of the communication system is divided into four types: positive normality(0), weak failure(1), intermediate failure(2), and complete failure(3). The intermediate failure state consists of two classes: State 1 and state 2. $a_{i j}(i, j=0,1,2,3)$ represents a transfer probability value between states, $O_{k}(k=0,1,2,3)$ representing the sequence of data observed in various states, that is, a fault symptom.

In most cases the choice is not important, and the B matrix is most important because it is directly related to the output observation symbol. State transfer probability array A can reflect process state sequence correlation information, which is important for some sequence-related modeling problems. In most cases, the initial parameter of $\mathrm{A}$ and $\pi$ is set to uniform distribution or non-zero random number. The communication system is always in normal working condition at the time of initial work or operation. The initial state probability parameter in the model parameter is set to $\pi=\left[\begin{array}{llll}1 & 0 & 0 & 0\end{array}\right]$.

Then the state transfer matrix could be described as follow:

$$
A=\left[\begin{array}{cccc}
a_{00} & a_{01} & 0 & 0 \\
0 & a_{11} & a_{12} & 0 \\
0 & 0 & a_{22} & a_{23} \\
0 & 0 & 0 & 1
\end{array}\right]
$$

It is shown that the model structure of the left-right HMM can reflect the irreversibility of the fault development and eventually develop into a complete fault.

Usually, the HMM model can be distinguished from the continuous HMM (CHMM)model and the discrete HMM
model(DHMM). In this paper, the discrete hidden Markov model is adopted, in view of the discrete characteristic of the circuit fault signal of the communication system. The biggest difference between DHMM and CHMM is parameter B. The B matrix of DHMM is a probability matrix. The observation data sequence is composed of a series of discrete vectors. For leftright HMM, it is not possible to train the model using a single observation sample sequence because the transient nature of the internal state of the model allows only a few observations for each state. So reliable model parameters need to be trained by multiple observation data sequences. The evaluation formula of Baum-Welch algorithm must be amended when using multiple observation sequences to train HMM. There are $\mathrm{K}$ order of observation as follows:

$$
O=\left[O^{(1)}, O^{(2)}, \cdots O^{(k)}\right]
$$

It indicates that $O^{(k)}=\left[O_{1}^{(k)}, O_{2}{ }^{(k)}, \cdots O_{T_{k}}{ }^{(k)}\right]$ is the length of the $\mathrm{K}$ observation sequence is generally set to equal length observation sequence, that is, all $T_{k}=T$, and requires that each observation sequence be independent of other observation sequences, and that:

$$
P(o \mid \lambda)=\prod_{k=1}^{K} P\left(O^{(k)} \mid \lambda\right)=\prod_{k=1}^{K} P_{K}
$$

The goal of model training is to adjust the parameters of the model $\lambda$ to maximum $P(o \mid \lambda)$. The reevaluation formula of multiple observation sequences can be obtained by summing up the probability of each observation sample. When training HMM using $\mathrm{L}$ observation sequences, the revaluation formula is amended to read:

$$
\left\{\begin{array}{l}
\bar{\pi}_{i}=\sum_{i=1}^{L} \alpha_{1, l}(i) \beta_{1, l}(i) / P\left(O_{l} \mid \lambda\right) \quad 1 \leq i \leq N \\
\bar{a}_{i j}=\frac{\sum_{l=1}^{L} \sum_{t=1}^{T_{l}-1} \alpha_{t, l}(i) a_{i j} b_{j}\left(O_{t+1, l}\right) \beta_{t+1, l}(j) / P\left(O_{l} \mid \lambda\right)}{\sum_{l=1}^{L} \sum_{t=1}^{T_{l}-1} \alpha_{t, l}(i) \beta_{t+1, l}(j) / P\left(O_{l} \mid \lambda\right)} \\
\bar{b}_{i k}=\frac{\sum_{l=1}^{L} \sum_{t=1 \cap O_{t}=V_{k}}^{T_{l}-1} \alpha_{t, l}(j) \beta_{t+1, l}(j) / P\left(O_{l} \mid \lambda\right)}{\sum_{l=1}^{L} \sum_{t=1}^{T_{l}-1} \alpha_{t, l}(j) \beta_{t+1, l}(j) / P\left(O_{l} \mid \lambda\right)}
\end{array}\right.
$$


Among them: $\alpha$ is forward variable, $\beta$ is backward variable, $L$ is observation sequence number, $T_{l}$ is each observation sequence length. The above formula is valid for DHMM, could play an important role for fault diagnosis of communication system.

\section{CONCLUSION}

From the preceding analysis, it can be seen that the HMM model is suitable for the application of communication system fault diagnosis. The three algorithms and fault prediction models presented in this paper are suitable for the communication system with typical electronic system signal characteristics. In order to improve the level of fault diagnosis and health management of communication system, the next step is to forecast the health status of communication system by combining with the life design of communication system.

\section{REFERENCES}

[1] Lisha Xia, Huajing Fang. HMM based modeling and health condition assessment for degradation process[C].Proceedings of the 25st Chinese Control and Decision Conference. Guiyang,China:2013:2945-2948

[2] Davis W,Hjsgaard S. Hidden Semi Markov Models for Multiple Observation Sequences[J].The mhsmm Package for R.Journal of Statiscal Software,2011(4):1-22

[3] AmjadyN,Shayanfar H A. Optimal placement of distributed generations considering voltage stability and power losses with observing voltagerelated constraints[J].Applied Energy,2014(11):1252-1260

[4] Lee J,Beal M. The infinite hidden markov model[M].Advances in neural information processinig systems,Cambridge,MA:MIT Press,2002:577586

[5] Nakano M,et al.Bayesian nonparametric spectrogram modeling based on infinite factorial infinite hidden markov model[C].Proceedings of the IEEE Workshop on Applications of Signal Processing to Audio and Acoustics,New Paltz,USA:2012,735-742

[6] Bin W,Yuegagn L. Rolling bearing faults diagnosis method based on HMM[C].Proceedings of IEEE International Conference on Measuring Technology and Mechatronics Automation,Changsha,China:2010,195198 